\title{
Factors of corticosteroid resistant nephrotic syndrome progression in children
}

\author{
Natalya Zhuravleva ${ }^{1,2^{*}}$, Vladimir Buryak ${ }^{1}$, Sergey Horoshev ${ }^{2}$, and Natalya Mineeva ${ }^{2}$ \\ ${ }^{1}$ Ural State Medical University, Faculty of Paediatrics, 620028 Ekaterinburg, Russian Federation \\ ${ }^{2}$ Regional Children's Clinical Hospital, Department of hospital paediatrics, 620149, Ekaterinburg, \\ Russian Federation
}

\begin{abstract}
Nephrotic syndrome (NS) is one of the most severe kidney pathologies. NS often relapses and becomes resistant to immunosuppressive therapy. In order to identify factors that prevent NS relapses development and possible increase of therapy effectiveness, we have analyzed the course nature of primary (idiopathic) and secondary frequently relapsing steroid-resistant nephrotic syndrome (FRNS) in 22 children who received immunosuppressive therapy (IS) (cyclosporin (CSA) or mycophenolic acid (MPA)). As a result of the study, the following factors of NS progression in observed children were revealed: hereditary predisposition for diseases of urinary system organs; presence of more than 5 small external abnormalities development, greater disease record, presence of herpetic infection and chronic infection focal points. In children with long disease experience, a reliably higher level of proteinuria was identified. At the time of examination, stage I chronic kidney disease was registered in most children, one in five children had stage II CKD. CKD stage was not dependent on the type of immunosuppressive therapy received $(p<0.05)$. For children who had NS activity persistence at the time of the study, it was recommended to re-perform nephrobiopsy in order to verify morphological changes in the kidneys, correction - including the institution of alternative immunosuppressive therapy.
\end{abstract}

\section{Introduction}

Nephrotic syndrome (NS) is one of the most severe kidney pathologies [1-3]. The annual incidence of nephrotic syndrome in child population is 2-7 cases per 100 thousand child population, prevalence $-12-16$ cases per 100 thousand $[4-5,7,8]$.

The classic signs of this disease pathology are well known, and treatment protocols have been developed. Despite this, nephrotic syndrome often relapses and becomes resistant to therapy. The cause remains unknown, but it is believed that idiopathic NS pathogenesis is associated with impaired regulation of the immune system, systemic circulating factors, or hereditary structural abnormalities of podocytes. Diagnostics and follow-up of FRNS in children is a big problem due to its heterogeneous etiology, frequent lack of remission in $10-30 \%$ of patients in carrying out prolonged immunosuppressive

\footnotetext{
*Corresponding author: jurnas178@yandex.ru
} 
therapy. FRNS is associated with an increased risk of terminal renal failure as well as relapse after kidney transplantation [5-12].

The following questions remain urgent: how to prevent disease recurrence, how to increase the effectiveness of therapy.

The purpose of the study was to analyze the progression factors, the course nature of primary (idiopathic) and secondary frequently relapsing steroid-resistant nephrotic syndrome (FRNS) in children receiving immunosuppressive therapy (IS) (cyclosporin (CSA) or mycophenolic acid (MPA)).

\section{Materials and Methods}

A retrospective analysis of patients' medical records (form 003/y) was carried out. The study has been conducted on the basis of the nephrological department of the state budgetary institution of health of Sverdlovsk region "Regional children's clinical hospital" of Yekaterinburg for two years: from 2017 to 2018 Medical records of 22 patients (form 003/y) admitted to the department in 2017 were retrospectively analyzed. In order to work with medical documentation and further publish the results of the conducted studies, informed written consent of the management of the regional children's clinical hospital was obtained.

The criteria for inclusion in the study group were: presence of frequently relapsing steroid-resistant nephrotic syndrome; morphological diagnosis; immunosuppressive therapy, ages 6 to 16. Criteria for exclusion were: inborn nephrotic syndrome, familial forms of nephrotic syndrome.

Result analysis was carried out using Statiatica 10.0 program. Methods of descriptive statistics were used. For the comparative analysis of parametric samples, the Strudent tcriterion, the non-parametric - U-criterion Mann - Whitney, the criterion $\chi 2$ were applied. Dependency analysis was performed using Pearson's linear correlation, Spearman's rank correlation, the results were considered reliable at a significance level of $\mathrm{p}<0.05$.

The work was carried out on the initiative of the physicians of the nephrological department of the state budgetary institution of health of Sverdlovsk region "Regional children's clinical hospital", employees of the Department of Hospital Pediatrics of the Ural State Medical University.

\section{Results and Discussion}

The study group consisted of $19(86.4 \%)$ boys and $3(13.6 \%)$ girls aged between 6 and 16 (average age $11.1 \pm 0.7$ years, Me-11.9 years, $\sigma-3 y$ ).

Analysis of the antenatal period in this group of children showed that there was an aggravated obstetric history in $81.8 \%(\mathrm{n}=18)$ cases - in most mothers $(54.5 \%, \mathrm{n}=12)$ pregnancy occurred with gestosis, $31.8 \%(\mathrm{n}=7)$ of mothers had infectious diseases, five mothers $(22.7 \%)$ had mild to moderate anemia. Pregnancy nephropathy and exacerbation of chronic pyelonephritis were observed in equal amounts — four (18.2\%) persons respectively. Delayed intrauterine development of the fetus was registered in $2(9.1 \%)$ cases. One (4.5\%) mother pregnancy was complicated by chronic fetoplacental insufficiency.

Most children were born from second and subsequent pregnancies - 63.6\% ( $n=14)$, from first pregnancy - 36.4\% $(n=8) .95 .5 \%(n=21)$ of babies were born in term, one $(4.5 \%)$ baby was premature. The average birth weight was $3336 \pm 75$ gr. ( $\sigma: 353.9$, Me:3385gr), body length $51.9 \pm 0.4 \mathrm{~cm}(\sigma: 2.0$. Me: $52 \mathrm{~cm})$. A higher number of children were breastfed up to nine months - $77.3 \%(n=17)$ of children; $13.6 \%(n=3)$ of children 
received early artificial feeding; $9.1 \%(n=2)$ of children received mixed feeding. The introduction of complementary food for all children was carried out in a timely manner.

Family history for diseases of the urinary system was weighed down in ten $(45.5 \%)$ children (chronic pyelonephritis, presence of congenital malformations of the urinary system), exchange diseases (urolithiasis, biliary disease) - in $3(13.6 \%)$ cases.

Physical development of children on immunosuppressive therapy in most cases $(n=18$, $81.8 \%$ ) corresponded to real age, lagged behind real age - in $13.6 \%(n=3)$ cases, exceeded - in one $(4,5 \%)$ case. The number of congenital small abnormal developments (CSAD) in examined children was 0 to 8 (in $31.8 \%(n=7)$ cases - CSAD $\geq 5$ ).

In the study group, idiopathic NS was observed in 18 (81.8\%) patients, secondary - in four $(18.2 \%)$ cases (on the phonenality of dysplasirenal tissue - in two $(9.1 \%)$ children, development of hemorrhagic vasculitis - in one (4.5\%) child, and hemolytic-uremic syndrome (STEC-HUS) - in one (4.5\%) child). Analysis of the genealogical history in examined patients allowed to exclude options with family NS.

In the structure of co-morbidities in this group of children, attention was paid to the persistence presence of the herpetic infection (EB infection, H6 infection, CMV infection) in the majority - $12(54.5 \%)$ patients; chronic infection focal points (chronic tonsillitis, rhinitis) - in $8(36.4 \%)$ patients; undifferentiated connective tissue dysplasia syndrome - in $16(72.8 \%)$ patients.

The average age of the disease onset was $5 \pm 0.6$ years $(\min -1.3 \mathrm{y}, \max -10.3, \mathrm{Me}-4.8 \mathrm{y}, \sigma-$ $2.3 \mathrm{y}$ ); disease duration at the time of examination - from 1 year 4 months to 12 years 3 months (average disease record - 5.8 \pm 0.6 years, Me-6.1y, $\sigma-2.7 y$ ). The leading factors in the nephrotic syndrome development in this group of children were: in most cases $(45.5 \%)$ - acute respiratory viral infection, in $2(9.1 \%)$ children - chronic tonsillitis exacerbation.

In the disease onset, complete nephrotic syndrome was observed in $81.8 \%(n=18)$ of cases with extrarenal manifestations (expressed peripheral $(81.8 \%)$ and cavitary $(18.2 \%)$ edema; arterial hypertension $(22.7 \%)$ ); proteinuria $(4.7 \pm 1.03 \mathrm{~g} /$ day); hypoproteinemia $(43.2 \pm 1.8 \mathrm{~g} / \mathrm{l})$; hypoalbuminemia $(21.8 \pm 1.5 \mathrm{~g} / \mathrm{l})$; hyperlipidemia in $63.6 \%(\mathrm{n}=14)$ of cases. The remaining $18.2 \%(n=4)$ of children had incomplete nephrotic syndrome (in the absence of edema). Asthenovegetative syndrome was typical for most children (68\%). Urinary syndrome in the form of microhematuria, leukocyturia, crystalluria $(36.4 \% ; 18.2 \% ; 4.5 \%$ respectively) was also present.

The disease record in the studied children with nephrotic syndrome on average was $5.9 \pm 0.6$ years: on MPA therapy $6.2 \pm 1.1$ years; CSA $-4.1 \pm 0.8$ years; $p>0.05$.

The average number of disease relapses was $5.9 \pm 0.6$, in children on MPA therapy $6.2 \pm 1.1$; on CA therapy $-4.1 \pm 0.8(\mathrm{p}>0.05)$. Over the last year, the average number of relapses was $0.9 \pm 0.3$ : in children on MPA therapy $-1.1 \pm 0.5$; on CA therapy $-0.7 \pm 0.24$ $(\mathrm{p}>0.05)$.

The duration of remission in patients of this group was from 2 months to 2.5 years: the maximum remission in children on MPA therapy was 2 years, on CSA - 2.5 years.

At the time of examination, most patients had persisting complete or partial clinical and laboratory remission $(86.4 \% ; n=19)$. Recurrence of activity was observed in three $(13.6 \%)$ children which showed peripheral edema, hypoproteinemia, hypoalbumyemia, hyperlipidemia; in one $(4,5 \%)$ case - oliguria. Asthenovegetative syndrome was typical for half of the children examined $(n=54.2 \%)$.

Proteinuria was absent in $36.4 \%$ of cases, distributed evenly in patients on IS therapy MPA and CSA. Minimum proteinuria persisted in half of children $(n=11)$ : in five $(22.7 \%)$ children who received MPA therapy and in six (27.3\%) children who were on CSA therapy. Pronounced proteinuria persists in two $(9.1 \%)$ children on MPA therapy and one $(4.5 \%)$ child on CSA therapy. 
Kidney function in children was retained in $22.7 \%(n=5)$ of cases. Partial impairment of kidney function was observed as: hyperfiltration (59.1\%); decreased filtration and concentration functions $(18.2 \%)$; hyperstenuria $(13.6 \%)$. The average glomerular filtration rate was $121.1 \pm 5.7 \mathrm{ml} / \mathrm{min} / 1.73 \mathrm{~m}^{2}$. Disruption of renal excretory function of mild degree according to the dynamic nephroscintigraphy was observed in six $(27.3 \%)$ children, average degree - in four $(18.2 \%)$ patients, severe degree - in one $(4.5 \%)$ child. No disorders of renal hemodynamics were detected according to the ultrasound dopplerography; vascularization of kidneys persisted in all the children examined.

According to the light and electron microscopy of nephrobioptate, diffuse mesangial proliferative glomerulonephritis $(81.2 \%)$ prevailed; nephrotic syndrome in diffuse membranose glomerulonephritis was registered in $13.6 \%(n=3)$ of cases, in one child $(4.5 \%)$ - NS with minimal changes (podocytopathy). Renal tissue dysplasia in the form of thin basal membranes was identified in $4(18.2 \%)$ patients. The tubulointerstitial component is minimal in $40.9 \%$ of patients, moderate in $27.3 \%(n=6)$ and pronounced in $9.1 \%(n=2)$ cases. Diffuse glomerulosclerosis areas were observed in two $(9.1 \%)$ cases; segmental glomerulosclerosis in one $(4.5 \%)$ child. Total glomerulosclerosis was verified in $6(27.3 \%)$ patients, with up to $75 \%$ of the glomerules sclerosed.

The criteria for prescribing immunosuppressive therapy in this group of patients were: frequently relapsing nephrotic syndrome observed in $17(77.3 \%)$ cases; steroid-resistant nephrotic syndrome - in five $(22.7 \%)$ children. At the time of the study, 11 (50\%) patients received Cyclosporin A therapy, and 11 (50\%) patients received Mycophenolic acid.

The average duration of therapy with mycophenolic acid was $2.2 \pm 0.5$ years, the average dose of the medication was $25.1 \pm 2.8 \mathrm{mg} / \mathrm{kg} /$ day. Among children of this group, most patients $(63.6 \%)$ changed immunosuppressive CSA therapy to MPA therapy in connection with the development of CSA-toxicity and CSA-dependence. The duration of CSA therapy in this patient group averaged $3.5 \pm 0.9$ years; the average dose of CSA was $3.3 \pm 0.5 \mathrm{mg} / \mathrm{kg}$. The reason for CSA therapy withdrawal in this group of children in two $(9.1 \%)$ cases was the manifestation of hepatotoxicity, in other cases - its insufficient effectiveness. Also, seven children $(63.6 \%)$ previously received therapy with cyclophosphamide, an average of $5.5 \pm 2.2$ courses before reaching the maximum permissible cumulative dose. The reasons for medication withdrawal were: in four cases $(57.1 \%)$ - inefficiency, in three cases $(13.6 \%)$ - development of leukopenia.

Against the background of immunosuppressive therapy, there was a reliable decrease in the level of daily proteinuria. The dynamics of proteinuria levels in observed patients is presented in Table 1.

Table 1. Dynamics of proteinuria level in observed patients depending on type of immunosuppressive therapy.

\begin{tabular}{|l|l|l|l|}
\hline $\begin{array}{l}\text { Type of immunosuppressive } \\
\text { therapy }\end{array}$ & $\begin{array}{l}\text { Proteinuria in disease } \\
\text { onset, g/day }\end{array}$ & $\begin{array}{l}\text { Proteinuria at the time } \\
\text { of study, g/day }\end{array}$ & $\mathrm{t}, \mathrm{p}$ \\
\hline Mycophenolic acid & $4.5 \pm 1.3$ & $1.53 \pm 0.87$ & $0.02 ; \mathrm{p}>0.5$ \\
\hline Cyclosporin A & $5.13 \pm 1.9$ & $0.96 \pm 0.65$ & $2.1 ; \mathrm{p}<0.05$ \\
\hline IS therapy in general & $4.72 \pm 1.03$ & $1.25 \pm 0.61$ & $2.9 ; \mathrm{p}<0.05$ \\
\hline
\end{tabular}

In multifactorial analysis, positive correlation between the following conditions was revealed: hereditary predisposition for diseases of urinary system organs, exchange pathology, presence of CSAD $>5$ and level of GFR (hyperfiltration) ( $p<0.05$; bonding force - medium $(C=0.236, p>0.05)$; presence of herpetic infection and chronic infection focal points and degree of proteinuria (bonding force $\mathrm{X} 2=0.37, \mathrm{C}=0.288, \mathrm{p}>0.05$ ). In children with long experience disease record, a reliably higher level of proteinuria $(t=2.37)$ was detected. At the time of examination, stage I chronic kidney disease was registered in most 
children $(81.8 \%)$, stage II CKD - in $18.2 \%$ of cases. CKD stage was not dependent on the type of immunosuppressive therapy received $(\mathrm{p}<0.05)$.

\section{Conclusion}

We revealed the following factors of NS progression in observed children were: hereditary predisposition for diseases of urinary system organs; more than five congenital small developmental abnormalities, greater disease record, herpetic infection and chronic infection focal points presence.

For children who had NS activity persistence at the time of the study, it was recommended to re-perform nephrobiopsy in order to verify morphological changes in the kidneys, correction - including the institution of immunosuppressive therapy (inc. alternative).

At the time of the research, most children (81.8\%) suffered from stage I chronic kidney disease; in $18.2 \%$ of cases - stage II CKD. CKD stage was not dependent on the type of immunosuppressive therapy received $(\mathrm{p}<0.05)$.

Identified risk factors of frequently relapsing steroid-resistant nephrotic syndrome substantiate the need for adequate therapy in manifestation of the disease, as well as early institution of immunosuppressive therapy.

\section{Acknowledgements}

We express our gratitude to Candidate of Medical Sciences Irina Evgenyevna Valamina for morphological verification of nephrotic syndrome in children.

\section{Conflict of interest}

The authors of this article confirmed the absence conflict of interests, financial or any other support which should be reported.

\section{References}

1. M. S. Ignatova, Nephrologist's handbook, (M.: «Overlej», 2016)

2. E. Lojmann, A. N. Cygin, A. A. Sarkisyan, Pediatrical nephrology: Practical guide, (Moscow: "Littera", 2010)

3. M. V. Matveeva, Optimization of immunosuppressive therapy with calcineurin inhibitors in children with nephrotic syndrome: candidate's thesis of Medical Science, 134 (Moscow, 2012)

4. V. R. Mello, M. T. Rodrigues, T. H. Mastrocinque, et al., Ped. Nephr, (2010)

5. A. Trautmann, M. Vivarelli, S. Samuel, et al., GUIDELINES IPNA Ped. Nephr, (2020)

6. N. V. Necheporenko, N. D. Savencova, N. M. Kalinina, Russian Bulletin of Perinatology and Pediatrics, 62 (2017)

7. P. A. Nyrkova, N. D. Savencova, Nephr, 19 (2015)

8. S. Dogra, F. Kaskel, Pediatric Nephrology, 6, 32 (2017)

9. G. Damien, N. Kazumoto Iijima, R. Parekh, Seminar, 392,10141 (2018)

K. Tullus, H. Webb, A. Bagga, Review, 2 (12) (2018)

11. A. Trautmann, S. Schnaidt, B. S. Lipska-Ziętkiewicz, et al., J.A.S.N., 28 (10), 2017

12. S. M. Samuel, R. Flynn, M. Zappitelli, et al., C.M.A.J. Open., 5 (2), 424-430 (2017) 